\title{
Acyclic Matching in Some Subclasses of Graphs
}

\author{
B. S. Panda ${ }^{(凶)}$ and Juhi Chaudhary \\ Computer Science and Application Group, Department of Mathematics, \\ Indian Institute of Technology Delhi, Hauz Khas 110016, New Delhi, India \\ bspanda@maths.iitd.ac.in, chaudhary.juhi5@gmail.com
}

\begin{abstract}
A subset $M \subseteq E$ of edges of a graph $G=(V, E)$ is called a matching if no two edges of $M$ share a common vertex. A matching $M$ in a graph $G$ is called an acyclic matching if $G[V(M)]$, the subgraph of $G$ induced by the $M$-saturated vertices of $G$ is acyclic. The ACYCLIC Matching Problem is the problem of finding an acyclic matching of maximum size. The decision version of the ACYCLIC MAtChing ProbLEM is known to be NP-complete for general graphs as well as for bipartite graphs. In this paper, we strengthen this result by showing that the decision version of the ACYCLIC Matching Problem remains NPcomplete for comb-convex bipartite graphs and dually-chordal graphs. On the positive side, we present linear time algorithms to compute an acyclic matching of maximum size in split graphs and proper interval graphs. Finally, we show that the ACyCLiC Matching Problem is hard to approximate within a factor of $n^{1-\epsilon}$ for any $\epsilon>0$, unless $P=N P$ and the Acyclic Matching Problem is APX-complete for $2 k+1$-regular graphs for $k \geq 3$, where $k$ is a constant.
\end{abstract}

Keywords: Matching $\cdot$ Bipartite graphs $\cdot$ Chordal graphs $\cdot$ Graph algorithm $\cdot \mathrm{NP}$-completeness $\cdot$ Approximation algorithm

\section{Introduction}

A subset $M \subseteq E$ of edges of a graph $G=(V, E)$ is called a matching if no two edges of $M$ share a common vertex. Vertices that are incident on the edges of a matching $M$ are called $M$-saturated vertices and are denoted by $V(M)$. In this paper, we study an important variant of matching called acyclic matching (see $[3,5,9]$ ). A matching $M$ in $G$ is called an acyclic matching if $G[V(M)]$, the subgraph of $G$ induced by the $M$-saturated vertices of $G$ is acyclic. The Acyclic Matching Problem asks to find an acyclic matching of maximum

B. S. Panda-The author thanks the SERB, Department of Science and Technology for their support vide Diary No. SERB/F/12949/2018-2019.

J. Chaudhary - The author has been supported by the Department of Science and Technology through INSPIRE Fellowship for this research.

(C) Springer Nature Switzerland AG 2020

L. Gąsieniec et al. (Eds.): IWOCA 2020, LNCS 12126, pp. 409-421, 2020.

https://doi.org/10.1007/978-3-030-48966-3_31 
size in a given graph $G$. The acyclic matching number of $G$, denoted by $\mu_{a c}(G)$ is the maximum size of an acyclic matching in $G$.

More formally, the ACYCLIC MATChing Problem and its decision version are defined as follows:

\section{Acyclic Matching Problem}

Instance: A graph $G=(V, E)$.

Solution: An acyclic matching $M$ in $G$.

Measure: Cardinality of the set $M$.

\section{Acyclic Matching Decide Problem}

Instance: A graph $G=(V, E)$ and a positive integer $k$.

Question: Does there exist an acyclic matching $M$ in $G$ of size at least $k$ ?

Goddard et al. [5] introduced the concept of acyclic matching along with some other variants of the matching and proved that the ACYCLIC MatchING Decide Problem is NP-complete for general graphs. Later, Panda and Pradhan [9] strengthened this result by showing that the ACYCLIC MatchING Decide Problem remains NP-complete for bipartite graphs and even for perfect-elimination bipartite graphs, which is a subclass of bipartite graphs. They also gave a dynamic programming based algorithm to find an acyclic matching of maximum size in bipartite permutation graphs. Baste et al. [2] showed that finding a maximum size 1-degenerate matching in a graph $G$ is equivalent to finding a maximum acyclic matching in $G$. They further proved that a maximum 1-degenerate matching could be found in polynomial time in chordal graphs, but the time complexity is very high. Recently, Fürst and Rautenbach showed that it is hard to decide whether a given bipartite graph of maximum degree at most four has a maximum matching that is acyclic [4]. They further characterized the graphs for which every maximum matching is acyclic and give linear time algorithms to compute a maximum acyclic matching in graph classes like $P_{4}$-free graphs and $2 P_{3}$-free graphs [4]. There are no approximation results known for the Acyclic Matching Problem till now.

In this paper, we study the complexity status of the ACYClic Matching Problem and the Acyclic Matching Decide Problem in some subclasses of graphs. The main contributions of this paper are summarized below.

1. We prove that the Acyclic Matching Decide Problem is NP-complete for tree-convex bipartite graphs by showing that it is NP-complete for combconvex bipartite graphs which is a subclass of tree-convex bipartite graphs.

2. We prove that the Acyclic Matching Decide Problem is NP-complete for dually chordal graphs.

3. We prove that a maximum size acyclic matching can be computed in linear time in split graphs and proper interval graphs.

4. We prove that it is hard to approximate the ACYClic Matching Problem within a factor of $n^{1-\epsilon}$ for any $\epsilon>0$, unless $P=N P$.

5. We prove that the ACYClic Matching Problem is APX-complete for $2 k+$ 1-regular graphs for $k \geq 3$, where $k$ is a constant. 


\section{Preliminaries}

We consider only simple and connected graphs. For a graph $G=(V, E)$, let $n$ denotes the number of vertices and $m$ denotes the number of edges in $G$. The open and closed neighborhood of a vertex $u \in V$ are denoted by $N(u)$ and $N[u]$ respectively, where $N(u)=\{w \mid w u \in E\}$ and $N[u]=N(u) \cup\{u\}$. The degree of a vertex $u$ is $|N(u)|$ and is denoted by $d(u)$. For a graph $G=(V, E)$, the subgraph of $G$ induced by $U \subseteq V$ is denoted by $G[U]$, where $G[U]=\left(U, E_{U}\right)$ and $E_{U}=\{x y \in E \mid x, y \in U\}$.

A graph $G=(V, E)$ is called a $k$-regular graph if $d(v)=k$ for every vertex $v$ of $G$. A graph $G=(V, E)$ is called a bipartite graph if its vertex set $V$ can be partitioned into two independent sets $X$ and $Y$, such that every edge of $G$ joins a vertex in $X$ to a vertex in $Y$. A comb is a graph obtained by attaching a pendant vertex (tooth) to every vertex of a path (backbone). A bipartite graph $G=(X, Y, E)$ is said to be a tree-convex bipartite graph, if a tree $T=\left(X, E_{X}\right)$ can be defined on $X$ such that for every vertex $y \in Y$, the vertices in $N_{G}(y)$ induces a subtree of $T$. It can be noted that tree-convex bipartite graphs are recognizable in linear time and the associated tree $T$ can also be constructed in linear time [11]. If the tree $T$ in a tree-convex bipartite graph is a comb, then $G$ is called a comb-convex bipartite graph.

A graph $G=(V, E)$ is called a chordal graph if every cycle in $G$ of length at least four has a chord, that is, an edge joining two non-consecutive vertices of the cycle. A graph $G=(V, E)$ is called a split graph if its vertex set $V$ can be partitioned into two sets $I$ and $C$ such that $I$ is an independent set and $C$ is a clique. Let $\mathscr{F}$ be a family of sets. The intersection graph of $\mathscr{F}$ is obtained by taking each set in $\mathscr{F}$ as a vertex and joining two sets in $\mathscr{F}$ if and only if they have a nonempty intersection. A graph $G$ is called a proper interval graph if it is the intersection graph of a family $\mathscr{F}$ of intervals on the real line such that no intervals in $\mathscr{F}$ contains another. A vertex $u \in N_{G}[v]$ in a graph $G$ is called a maximum neighbor of $v$ if for all $w \in N_{G}[v], N_{G}[w] \subseteq N_{G}[u]$. An ordering $\alpha=\left(v_{1}, v_{2}, \ldots, v_{n}\right)$ of $V(G)$ is called a maximum neighborhood ordering, if $v_{i}$ has a maximum neighbor in $G_{i}=G\left[\left\{v_{i}, \ldots, v_{n}\right\}\right]$ for all $i, 1 \leq i \leq n$. A graph $G$ is called a dually chordal graph if it has a maximum neighborhood ordering.

\section{NP-Completeness Results}

\subsection{Comb-Convex Bipartite Graphs}

It has been shown in [9] that the Acyclic Matching Decide Problem is NPcomplete for bipartite graphs. In this subsection, we strengthen this result by showing that the ACYCLIC Matching Decide Problem remains NP-complete for tree-convex bipartite graphs, which is a subclass of bipartite graphs by showing that it is NP-complete for comb-convex bipartite graphs.

Theorem 1. The Acyclic Matching Decide Problem is NP-complete for comb-convex bipartite graphs. 
Proof. Clearly, the Acyclic Matching Decide Problem belongs to the class NP for comb-convex bipartite graphs. To show the NP-completeness, we give a polynomial reduction from the Acyclic Matching Decide Problem for bipartite graphs, which is already known to be NP-complete [9].

Given a bipartite graph $G=(X, Y, E)$, we construct a comb-convex bipartite graph $H=\left(X_{H}, Y_{H}, E_{H}\right)$ as follows:

Let $X_{H}=X \cup X^{\prime}$, where $X^{\prime}=\left\{x_{i}^{\prime} \mid x_{i} \in X\right\}, Y_{H}=Y$, and $E_{H}=E \cup E^{\prime}$, where $E^{\prime}=\left\{x_{i}^{\prime} y \mid x_{i}^{\prime} \in X^{\prime}\right.$ and $\left.y \in Y\right\}$. The constructed graph $H$ is a combconvex bipartite graph if $X^{\prime}$ is taken as the backbone and $X$ is taken as the teeth of a comb $C$. Further, note that given a bipartite graph $G$, the graph $H$ can be constructed in polynomial time.

Now, the following claim is sufficient to complete the proof of the theorem.

Claim. $G$ has an acyclic matching of size at least $k$ if and only if $H$ has an acyclic matching of size at least $k$.

Proof. Necessity: Let $M$ be an acyclic matching in $G$ of size at least $k$. Since $G$ is a vertex induced subgraph of graph $H, M$ is acyclic in $H$. Hence, $H$ has an acyclic matching of size at least $k$.

Sufficiency: Let $M^{\prime}$ be an acyclic matching in $H$ of size at least $k$. If $M^{\prime}$ does not have an edge from the edge set $E^{\prime}$ then $M^{\prime}$ is a required acyclic matching in $G$. Otherwise, note that $M^{\prime}$ can include at most one edge from the edge set $E^{\prime}$. To the contrary, if $\left\{x_{i}^{\prime} y_{i}, x_{j}^{\prime} y_{j}\right\} \subseteq M^{\prime}$ for some $x_{i}^{\prime}, x_{j}^{\prime} \in X^{\prime}$ then $G\left[\left\{x_{i}^{\prime}, y_{i}, x_{j}^{\prime}, y_{j}\right\}\right]$ forms a cycle, which is a contradiction. Thus, $M^{\prime}$ can include at most one edge from $E^{\prime}$.

Next, let $x_{i}^{\prime} y_{i} \in M^{\prime}$ for some $x_{i}^{\prime} \in X^{\prime}$. Since $G$ is connected, $y_{i}$ will have a neighbor (say $x_{k}$ ) in $X$. Note that $x_{k}$ must be unsaturated by $M^{\prime}$ because otherwise if $x_{k} y_{k} \in M^{\prime}$ for some $y_{k} \in Y$, then $G\left[\left\{x_{k}, y_{k}, x_{i}^{\prime}, y_{i}\right\}\right]$ will form a cycle, which is a contradiction to the fact that $M^{\prime}$ is acyclic. Let $M=\left(M^{\prime} \backslash\left\{x_{i}^{\prime} y_{i}\right\}\right) \cup$ $\left\{x_{k} y_{i}\right\}$. If $G[V(M)]$ is acyclic, then $M$ is a required acyclic matching in $G$. Otherwise, let us assume that $G[V(M)]$ contains a cycle $C^{\prime}$. If $C^{\prime}$ does not contain the vertex $x_{k}$, then $C^{\prime}$ is also a cycle in $G\left[V\left(M^{\prime}\right)\right]$. This contradicts the fact that $M^{\prime}$ is an acyclic matching. So, $C^{\prime}$ contains the vertex $x_{k}$. Let $x_{k} y_{a}, x_{k} y_{b} \in E\left(C^{\prime}\right)$. Since vertices of set $X^{\prime}$ are adjacent to every $y \in Y, x_{i}^{\prime} y_{a}, x_{i}^{\prime} y_{b} \in E_{H}$. Now, $C=\left(C^{\prime} \backslash\left\{x_{k} y_{a}, x_{k} y_{b}\right\}\right) \cup\left\{x_{i}^{\prime} y_{a}, x_{i}^{\prime} y_{b}\right\}$ is also a cycle in $G\left[V\left(M^{\prime}\right)\right]$, which is a contradiction. Hence, $M$ is acyclic and it is a required acyclic matching in $G$ of size at least $k$.

Hence, the Acyclic Matching Decide Problem is NP-complete for comb-convex bipartite graphs.

Corollary 1. The Acyclic Matching Decide Problem is NP-complete for tree-convex bipartite graphs.

\subsection{Dually Chordal Graphs}

The Acyclic Matching Problem is polynomial time solvable for chordal graphs [2] and hence for strongly chordal graphs. In this subsection, we show 
that the Acyclic Matching Decide Problem is NP-complete for dually chordal graphs which is a superclass of strongly chordal graphs.

Theorem 2. The Acyclic Matching Decide Problem is NP-complete for dually chordal graphs.

Proof. Clearly, the Acyclic Matching Decide Problem belongs to the class NP for dually chordal graphs. To show the NP-completeness, we give a polynomial reduction from the Acyclic Matching Decide Problem for general graphs, which is already known to be NP-complete [5].

Given a graph $G=(V, E)$, we construct a dually chordal graph $H=$ $\left(V_{H}, E_{H}\right)$ as follows: $V_{H}=V \cup\left\{v_{0}\right\}, E_{H}=E \cup\left\{v_{0} v \mid v \in V\right\}$.

Consider the ordering $\alpha=\left(v_{1}, v_{2}, \ldots, v_{n}, v_{0}\right)$. Since $N\left[v_{j}\right] \subseteq N\left[v_{0}\right]$ for all $v_{j} \in V, v_{0}$ is a maximum neighbor for all $v_{j}$ in $G_{j}=G\left[\left\{v_{j}, \ldots, v_{n}\right\}\right]$. Therefore, it is easy to see that the constructed graph $H=\left(V_{H}, E_{H}\right)$ is a dually chordal graph. Also, note that given a graph $G$, the graph $H$ can be constructed in polynomial time.

Now, the following claim is sufficient to complete the proof of the theorem.

Claim. $G$ has an acyclic matching of size at least $k$ if and only if $H$ has an acyclic matching of size at least $k$, where $k>1$.

Proof. Necessity: Let $M$ be an acyclic matching in $G$ of size at least $k$. Since $G$ is a vertex induced subgraph of $H$, so $M$ is an acyclic matching in graph $H$ of size at least $k$.

Sufficiency: Let $M^{\prime}$ be an acyclic matching in graph $H$ of size at least $k$, $k>1$. Observe that if the vertex $v_{0}$ is saturated by $M^{\prime}$, that is $v_{0} v_{i} \in M^{\prime}$ for some $v_{i} \in V$, then $\left|M^{\prime}\right|=1$. To the contrary, if there exists another edge $v_{j} v_{k} \in M^{\prime}$, then the graph $H\left[\left\{v_{0}, v_{j}, v_{k}\right\}\right]$ forms a cycle, which is a contradiction.

As $\left|M^{\prime}\right| \geq k>1$, vertex $v_{0}$ is not saturated by $M^{\prime}$, that is, $M^{\prime}$ does not have any edge of the form $v_{0} v_{i}$ for any $v_{i} \in V$. Thus, $M^{\prime}$ is a required acyclic matching in graph $G$ of size at least $k$.

Hence, the Acyclic Matching Decide Problem is NP-complete for dually chordal graphs.

\section{Polynomial Time Algorithms}

\subsection{Split Graphs}

In this subsection, we show that an acyclic matching of maximum size can be computed in linear time for split graphs which is a subclass of chordal graphs, where the complexity of computing a maximum size acyclic matching is $O\left(n^{7}\right)$.

Let $G=(V, E)$ be a split graph. Throughout this section, $I \cup C$ represents a given partition of the vertex set $V$, where $I$ is an independent set and $C$ is a clique in $G$. Now, the following lemma shows that the cardinality of an acyclic matching in a split graph $G=(V, E)$ can be either 1 or 2 only. 
Lemma 1. Let $G=(V, E)$ be a split graph. If $M$ is an acyclic matching in $G$, then $1 \leq|M| \leq 2$.

Proof. Let $M$ be an acyclic matching in $G$ and let $|M| \geq 3$. Let $\left\{a_{1} b_{1}, a_{2} b_{2}\right.$, $\left.a_{3} b_{3}\right\} \subseteq M$ for some $a_{i}, b_{i} \in V, 1 \leq i \leq 3$. Since $I$ is an independent set, we can assume without loss of generality that $b_{1}, b_{2}, b_{3} \in C$. This leads to a contradiction as $G\left[\left\{b_{1}, b_{2}, b_{3}\right\}\right]$ forms a cycle. Thus, $|M| \leq 2$.

Next, we will characterize the split graphs depending on the size of an acyclic matching in $G$. For this purpose, let us recall the definition of threshold graphs, which is a proper subclass of split graphs.

A split graph $G=(V, E)$ is called a threshold graph if the vertices in $I$ can be linearly ordered, say $\left(v_{1}, v_{2}, \ldots v_{|I|}\right)$, such that $N\left(v_{1}\right) \subseteq N\left(v_{2}\right) \subseteq \ldots \subseteq N\left(v_{|I|}\right)$. This linear ordering of a threshold graph can be computed in linear time [6].

Lemma 2. Let $G=(V, E)$ be a split graph and let $M$ be a maximum acyclic matching in $G$. Then, $|M|=2$ if and only if there exist a pair of vertices $v_{i}, v_{j} \in I$ such that $N\left(v_{i}\right) \backslash N\left(v_{j}\right) \neq \emptyset$ and $N\left(v_{j}\right) \backslash N\left(v_{i}\right) \neq \emptyset$.

Proof. Necessity: Let $M$ be an acyclic matching in $G$ and let $M=\left\{a_{i} b_{i}, a_{j} b_{j}\right\}$. Since $C$ is a clique and $I$ is an independent set, exactly two vertices from the set $\left\{a_{i}, a_{j}, b_{i}, b_{j}\right\}$ belong to $C$ and the other two belongs to $I$. Without loss of generality, let us assume that $a_{i}, a_{j} \in C$ and $b_{i}, b_{j} \in I$. Since $G\left[\left\{a_{i}, a_{j}, b_{i}, b_{j}\right\}\right]$ is acyclic and $a_{i} b_{i}, a_{j} b_{j}, a_{i} a_{j} \in E$, so $a_{i} \notin N\left(b_{j}\right)$ and $a_{j} \notin N\left(b_{i}\right)$. Hence, $b_{i}, b_{j} \in I$ is the required pair of vertices.

Sufficiency: Let us assume that there exist two vertices $v_{1}, v_{2} \in I$ such that $N\left(v_{1}\right) \backslash N\left(v_{2}\right) \neq \emptyset$ and $N\left(v_{2}\right) \backslash N\left(v_{1}\right) \neq \emptyset$. Let $c_{1} \in N\left(v_{1}\right) \backslash N\left(v_{2}\right)$ and $c_{2} \in$ $N\left(v_{2}\right) \backslash N\left(v_{1}\right)$. Now, define a matching $M=\left\{v_{1} c_{1}, v_{2} c_{2}\right\}$. It is easy to see that $G\left[\left\{v_{1}, c_{1}, v_{2}, c_{2}\right\}\right]$ contains no cycle, and hence $M$ is acyclic.

Theorem 3. Let $G=(V, E)$ be a split graph and let $M$ be a maximum acyclic matching in $G$. Then, $|M|=1$ if and only if $G$ is a threshold graph.

Proof. Necessity: Let $G=(V, E)$ be a split graph and let $M$ be a maximum acyclic matching in $G$ such that $|M|=1$. For the sake of contradiction, let us suppose that $G$ is not a threshold graph. Then, there will exist a pair of vertices $v_{i}, v_{j} \in I$ such that $N\left(v_{i}\right) \nsubseteq N\left(v_{j}\right)$ and $N\left(v_{j}\right) \nsubseteq N\left(v_{i}\right)$. Define a matching $M^{\prime}=\left\{c_{i} v_{i}, c_{j} v_{j}\right\}$, where $c_{i} \in N\left(v_{i}\right) \backslash N\left(v_{j}\right)$ and $c_{j} \in N\left(v_{j}\right) \backslash N\left(v_{i}\right)$. It is easy to see that $M^{\prime}$ is acyclic as $G\left[\left\{v_{i}, v_{j}, c_{i}, c_{j}\right\}\right]$ is a path graph. Since $\left|M^{\prime}\right|=2$, this leads to a contradiction to the fact that $M$ is a maximum acyclic matching in $G$. Hence, $G$ is a threshold graph.

Sufficiency: Let $G=(V, E)$ be a threshold graph and let $\left(v_{1}, v_{2}, \ldots v_{|I|}\right)$ be an ordering of $I$, such that $N\left(v_{1}\right) \subseteq N\left(v_{2}\right) \subseteq \ldots \subseteq N\left(v_{|I|}\right)$. Clearly, there does not exist any pair of vertices $v_{i}, v_{j} \in I$ such that $N\left(v_{i}\right) \backslash N\left(v_{j}\right) \neq \emptyset$ and $N\left(v_{j}\right) \backslash N\left(v_{i}\right) \neq \emptyset$. Hence, by Lemma 1 and Lemma 2, it is easy to see that $|M|=1$.

Based on the above discussions we have the following theorem.

Theorem 4. A maximum size acyclic matching in a split graph $G=(V, E)$ can be computed in $O(|V|+|E|)$ time. 
Proof. Due to space restriction, the proof has been deferred to the longer version of the paper.

\subsection{Proper Interval Graphs}

In this subsection, we show that an acyclic matching of maximum size can be computed in linear time for proper interval graphs which is a subclass of chordal graphs, where the complexity of computing a maximum size acyclic matching is $O\left(n^{7}\right)$.

Let $G=(V, E)$ be a given graph. A vertex $v \in V$ is called a simplicial vertex, if $N[v]$ induces a clique in $G$. An ordering $\alpha=\left(v_{1}, v_{2}, \ldots, v_{n}\right)$ of vertices is called a perfect elimination ordering (PEO) of $G$ if $v_{i}$ is a simplicial vertex in $G_{i}=$ $G\left[\left\{v_{i}, v_{i+1}, \ldots, v_{n}\right\}\right]$ for all $1 \leq i \leq n$. A PEO $\alpha=\left(v_{1}, v_{2}, \ldots, v_{n}\right)$ of a graph $G$ is called a bi-compatible elimination ordering $(\mathrm{BCO})$ if $\alpha^{-1}=\left(v_{n}, v_{n-1}, \ldots, v_{1}\right)$ i.e., the reverse of $\alpha$, is also a PEO of $G$. It has been characterized in [7] that a graph is proper interval if and only if it has a BCO.

Observation 5. [8] Let $\sigma=\left(v_{1}, v_{2}, \ldots, v_{n}\right)$ be a BCO of a proper interval graph $G$. If $v_{i} v_{j} \in E$, then $v_{k} v_{j} \in E$ for all $k, i \leq k \leq j-1$.

Observation 6. Let $\sigma=\left(v_{1}, v_{2}, \ldots, v_{n}\right)$ be a $\mathrm{BCO}$ of a proper interval graph $G$ and let $L\left[v_{i}\right]$ denotes the last neighbor of a vertex $v_{i}$ in $\sigma$. If $v_{i}<v_{j}$ in $\sigma$, then $L\left[v_{i}\right] \leq L\left[v_{j}\right]$.

Proof. Let us suppose that there exists $v_{i}$ and $v_{j}$ such that $v_{i}<v_{j}$ in $\sigma$ and $L\left[v_{i}\right]>L\left[v_{j}\right]$. Then by Observation $5, v_{j} L\left[v_{i}\right] \in E$ but since $L\left[v_{j}\right]<L\left[v_{i}\right]$, we reach at a contradiction.

Observation 7. Let $\sigma=\left(v_{1}, v_{2}, \ldots, v_{n}\right)$ be a $\mathrm{BCO}$ of a proper interval graph $G$ and let $L\left[v_{i}\right]$ denotes the last neighbor of a vertex $v_{i}$ in $\sigma$. If $M$ is an acyclic matching in $G$, then at most two vertices from the set $\left\{v_{i}, v_{i+1}, \ldots, L\left[v_{i}\right]\right\}$ can be saturated by $M$.

Proof. The result easily follows from Observation 5.

Lemma 3. Let $G$ be a proper interval graph with a $\mathrm{BCO} \sigma=\left(v_{1}, v_{2}, \ldots, v_{n}\right)$ and let $M$ be an acyclic matching in $G$. If the edges $u_{1} w_{1}, u_{2} w_{2} \in M$ such that $u_{1}<w_{1}$ and $u_{2}<w_{2}$ in $\sigma$, then either $w_{1}<u_{2}$ or $w_{2}<u_{1}$.

Proof. Let us assume without loss of generality that there exist two edges $e_{1}=$ $u_{1} w_{1}$ and $e_{2}=u_{2} w_{2}$ such that $u_{1}<w_{2}<w_{1}$ in $\sigma$. Now, the $G\left[\left\{u_{1}, w_{2}, w_{1}\right\}\right]$ forms a cycle, which is a contradiction. Thus, either $w_{1}<u_{2}$ or $w_{2}<u_{1}$.

Lemma 4. Let $G$ be a proper interval graph with a $\mathrm{BCO} \sigma=\left(v_{1}, v_{2}, \ldots, v_{n}\right)$ and let $M$ be a maximum acyclic matching in $G$. Then, there exists an acyclic matching $M^{\prime}$ in $G$ such that $v_{1} v_{2} \in M^{\prime}$ and $\left|M^{\prime}\right|=|M|$.

Proof. Let $G$ be a proper interval graph with a $\mathrm{BCO} \sigma=\left(v_{1}, v_{2}, \ldots, v_{n}\right)$ and let $M$ be a maximum acyclic matching in $G$. Let $v_{a} v_{b}$ be the first edge with respect to $\sigma$ that belongs to $M$. Let us assume without loss of generality that 
$v_{a}<v_{b}$. It is easy to see that $v_{a} \leq L\left[v_{2}\right]$ in $\sigma$. To the contrary, if $v_{a}>L\left[v_{2}\right]$ in $\sigma$, then $G\left[\left\{v_{1}, v_{2}, v_{a}, v_{b}\right\}\right]$ is acyclic and hence the edge $v_{1} v_{2}$ can be added to $M$. This leads to a contradiction to the fact that $M$ is a maximum acyclic matching in $G$. Hence, $v_{a} \leq L\left[v_{2}\right]$ in $\sigma$. Now, if $v_{a}=v_{2}$, then replace $v_{2} v_{b}$ by $v_{2} v_{1}$ in $M$. If $v_{a} \neq v_{2}$, then replace $v_{a} v_{b}$ by $v_{a} v_{2}$ in $M$. If $v_{a}=v_{1}$, then we are done. Otherwise, again replace the edge $v_{a} v_{2}$ by $v_{1} v_{2}$ in $M$.

By Observation 6 and Lemma 3, it is easy to see that we can replace the edge $v_{a} v_{b}$ with the desired edges in the cases mentioned above.

Let $\sigma=\left(v_{1}, v_{2}, \ldots, v_{n}\right)$ be a $\mathrm{BCO}$ of a proper interval graph $G$ and let $\sigma^{\prime}=\left(v_{a}, v_{b}, \ldots, v_{k}\right)$ be an ordering obtained from $\sigma$ by removing some vertices from $\sigma$. Then, $\sigma^{\prime}$ is also a BCO of some proper interval graph $G^{\prime}$, where $G^{\prime}$ is a subgraph of $G$. Hence, we have the following corollary to Lemma 4.

Corollary 2. If $\sigma^{\prime}=\left(v_{a}, v_{b}, \ldots, v_{k}\right)$ is a $\mathrm{BCO}$ of a subgraph $G^{\prime}$ of a proper interval graph $G$, then the edge $v_{a} v_{b}$ is contained in some maximum acyclic matching of $G^{\prime}$.

Based on the above lemmas, we now present a linear time algorithm AM$\operatorname{PIG}(G)$, which computes an acyclic matching of maximum size in a given proper interval graph $G$. The pseudocode of the algorithm is given below:

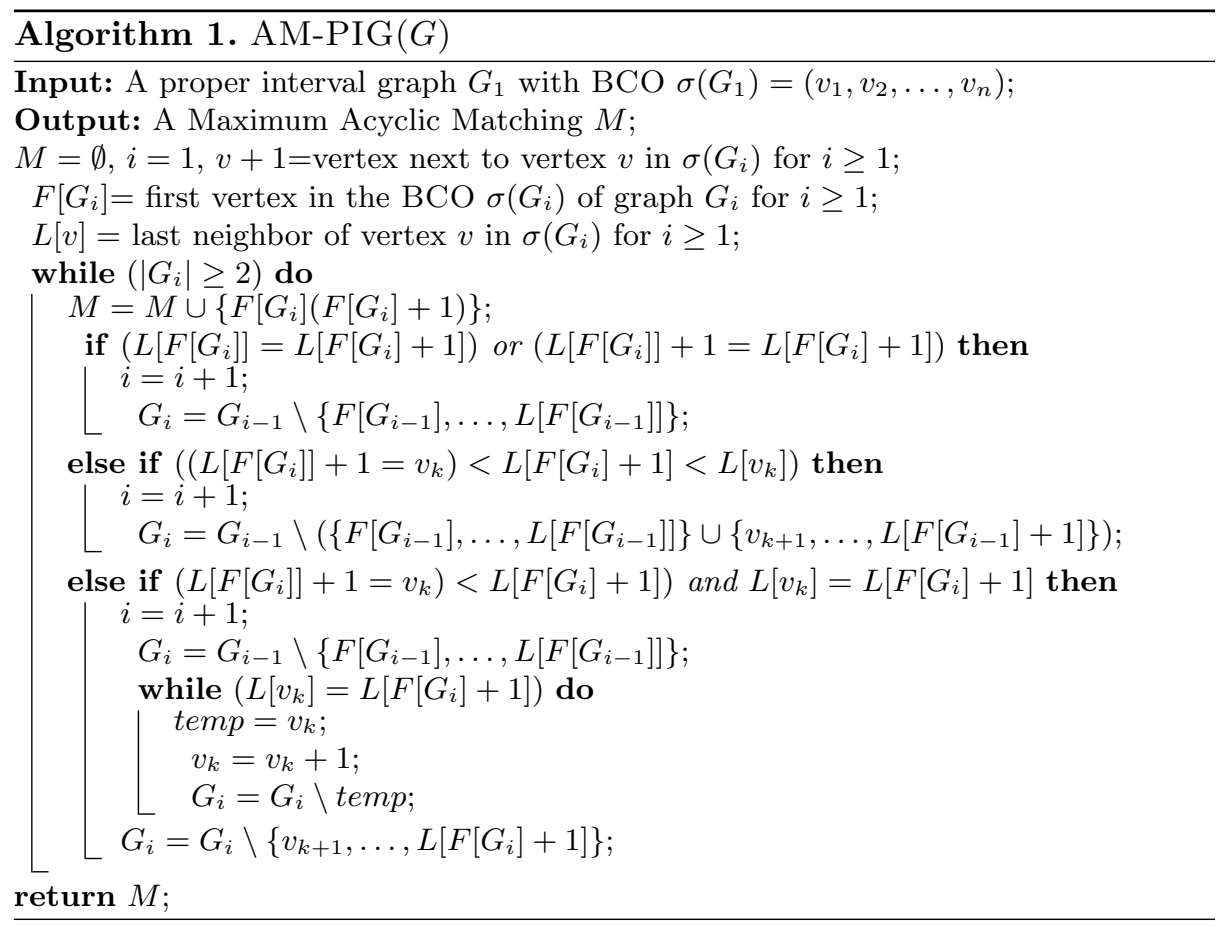

Theorem 8. Given a proper interval graph $G_{1}$ with $\mathrm{BCO} \sigma\left(G_{1}\right)$, AM$\operatorname{PIG}\left(G_{1}\right)$ correctly computes a maximum size acyclic matching in $G_{1}$. 
Proof. Due to space restriction, the proof has been deferred to the longer version of the paper.

\section{Inapproximation Results}

Let $G=(V, E)$ be a graph with $n$ vertices. It is easy to note that the maximum size of an acyclic matching in $G$ can be at most $\frac{n}{2}$. So, the ACYClic Matching Problem can be approximated within a factor of $n$ in polynomial time. In this section, we show that for any $\epsilon>0$, it is hard to approximate the ACYCLIC Matching Problem within a factor of $n^{1-\epsilon}$, unless $P=N P$.

To prove the result, we will need the following theorem for the MAXIMUM INDEPENDENT SET PROBLEM.

Theorem 9. [12] The Maximum Independent Set Problem for a graph G cannot be approximated within a factor of $n^{1-\epsilon}$ for any $\epsilon>0$, unless $P=N P$.

Now, consider the following construction:

Construction 1. Let $G=(V, E)$, where $V=\left\{v_{1}, v_{2}, \ldots, v_{n}\right\}$, be an instance of the Maximum Independent Set Problem. We construct a graph $H=$ $\left(V_{H}, E_{H}\right)$, an instance of the Acyclic Matching Problem, in the following way:

- $V_{H}=V \cup V^{\prime}$, where $V^{\prime}=\left\{v_{i}^{\prime} \mid v_{i} \in V\right\}$.

- $E_{H}=E \cup\left\{v_{i} v_{i}^{\prime} \mid 1 \leq i \leq n\right\} \cup\left\{v_{i} v_{j}^{\prime} \mid v_{i} v_{j} \in E\right\} \cup\left\{v_{i}^{\prime} v_{j}^{\prime} \mid v_{i} v_{j} \in E\right\}$.

Clearly, $H$ can be constructed in polynomial time as $\left|V_{H}\right|=2|V|$ and $\left|E_{H}\right|=$ $4|E|+|V|$.

Also, note that the edges in $H$ can be one of the following four types:

1. Type- $I=\left\{v_{i} v_{i}^{\prime} \mid v_{i} \in V\right.$ and $\left.v_{i}^{\prime} \in V^{\prime}\right\}$.

2. Type-II $=\left\{v_{i} v_{j} \mid v_{i}, v_{j} \in V\right\}$.

3. Type- $I I I=\left\{v_{i}^{\prime} v_{j} \mid v_{i}^{\prime} \in V^{\prime}\right.$ and $\left.v_{j} \in V\right\}$.

4. Type- $I V=\left\{v_{i}^{\prime} v_{j}^{\prime} \mid v_{i}^{\prime}, v_{j}^{\prime} \in V^{\prime}\right\}$.

Now, we will discuss some lemmas that will be used in the proof of the main theorem of this section. Let us recall that $V_{H}(M)$ denotes the set of $M$-saturated vertices of graph $H$.

Lemma 5. Let $H$ be the graph obtained from a given graph $G$ by Construction 1. If $M$ is an acyclic matching in $H$, then there exists an acyclic matching $M^{\prime}$ in $H$ such that $\left|M^{\prime}\right|=|M|$ and $M^{\prime}$ contains edges of Type-I and Type-II only.

Proof. First, let us suppose that $M$ is an acyclic matching in $H$ and let $M$ contains an edge (say $v_{i}^{\prime} v_{j}^{\prime}$ ) of Type- $I V$. Since $M$ is acyclic and $v_{i}^{\prime} v_{j}^{\prime} \in M$, both $v_{i}$ and $v_{j}$ are unsaturated by $M$. Let $M^{\prime}=\left(M \backslash\left\{v_{i}^{\prime} v_{j}^{\prime}\right\}\right) \cup\left\{v_{i}^{\prime} v_{j}\right\}$. If $G\left[V_{H}\left(M^{\prime}\right)\right]$ is acyclic, then we are done. So, assume that $G\left[V_{H}\left(M^{\prime}\right)\right]$ contains a cycle $C$. If $C$ does not contain the vertex $v_{j}$, then $C$ is also a cycle in $G\left[V_{H}(M)\right]$. This 
contradicts the fact that $M$ is an acyclic matching. So, $C$ contains the vertex $v_{j}$. Let $v_{j} u_{a}, v_{j} u_{b} \in E(C)$. Since $N\left[v_{j}\right]=N\left[v_{j}^{\prime}\right], v_{j}^{\prime} u_{a}, v_{j}^{\prime} u_{b} \in E_{H}$. Now, $C^{\prime}=\left(C \backslash\left\{v_{j} u_{a}, v_{j} u_{b}\right\}\right) \cup\left\{v_{j}^{\prime} u_{a}, v_{j}^{\prime} u_{b}\right\}$ is a cycle in $G\left[V_{H}(M)\right]$, which is a contradiction. Hence, $M^{\prime}$ is acyclic. In this way, an acyclic matching of same size can be obtained by replacing an edge of Type- $I V$ with a corresponding Type-III edge.

Using the similar arguments, we can show that an acyclic matching of same size can be obtained by replacing an edge of Type-III with a corresponding Type-II edge.

Lemma 6. Let $H$ be the graph obtained from a given graph $G$ by Construction 1 . If $M^{\prime}$ is an acyclic matching in $H$ containing edges of Type-I and Type-II only, then there exists an acyclic matching $M^{\prime \prime}$ in $H$ such that $\left|M^{\prime \prime}\right|=\left|M^{\prime}\right|$ and $M^{\prime \prime}$ contains edges of Type-I only.

Proof. Due to space restriction, the proof has been deferred to the longer version of the paper.

The following lemma shows that the described reduction is exactly what we need.

Lemma 7. Let $H$ be the graph obtained from a given graph $G$ by Construction 1 . Then, $G$ has an independent set of size at least $k$ if and only if $H$ has an acyclic matching of size at least $k$.

Proof. Necessity: Let $I=\left\{v_{1}, v_{2}, \ldots, v_{l}\right\}$ be an independent set in $G$ of size at least $k$. Define a matching $M=\left\{v_{1} v_{1}^{\prime}, v_{2} v_{2}^{\prime}, \ldots, v_{l} v_{l}^{\prime}\right\}$ in $H$. It is easy to see that $M$ is an acyclic matching as $G[V(M)]$ is a disjoint union of $K_{2}^{\prime} s$.

Sufficiency: Let $M=\left\{e_{1}, e_{2}, \ldots, e_{l}\right\}$ be an acyclic matching in $H$ of size at least $k$. By Lemma 6, there exists an acyclic matching $M^{\prime}$ in $H$ such that $\left|M^{\prime}\right|=$ $|M|$ and $M^{\prime}$ contains edges of Type- $I$ only. Define a set $I=\left\{v_{i} \mid v_{i} v_{i}^{\prime} \in M^{\prime}\right\}$. It is easy to see that $I$ is an independent set of graph $G$.

Corollary 3. G has a maximum independent set of size $k$ if and only if $H$ has a maximum acyclic matching of size $k$.

Theorem 10. The ACYClic MAtching Problem for a graph $G$ cannot be approximated within a factor of $n^{1-\epsilon}$ for any $\epsilon>0$, unless $P=N P$.

Proof. Let $G=(V, E)$ be a graph with $n$ vertices. Construct a graph $H=$ $\left(V_{H}, E_{H}\right)$ with $\left|V_{H}\right|=\bar{n}$ from $G$ using Construction 1. Let $I^{*}$ denotes a maximum independent set in $G$ and $M^{*}$ denotes a maximum acyclic matching in $H$.

Now, let us suppose that the ACYClic MATChing Problem can be approximated within a ratio $\alpha \geq 1$ by using an algorithm $A L G$, where $\alpha=\bar{n}^{1-\epsilon^{\prime}}$ for some fixed $\epsilon^{\prime}>0$.

If $M_{A L G}(H)$ is an acyclic matching in $H$ obtained by applying algorithm ALG, then $\left|M^{*}(H)\right| \leq \alpha\left|M_{A L G}(H)\right|$. 
By Corollary 3, $\left|I^{*}(G)\right|=\left|M^{*}(H)\right|$. By Lemma 7, we can construct an independent set $I_{A L G}$ of $G$ corresponding to $M_{A L G}$ of $H$ such that $\left|M_{A L G}(H)\right|=$ $\left|I_{A L G}(G)\right|$.

Hence, we obtain, $\left|I^{*}(G)\right| \leq \alpha\left|I_{A L G}(G)\right|=\bar{n}^{1-\epsilon^{\prime}}\left|I_{A L G}(G)\right|=(2 n)^{1-\epsilon^{\prime}} \mid I_{A L G}$ $(G)\left|=(2)^{1-\epsilon^{\prime}}(n)^{1-\epsilon^{\prime}}\right| I_{A L G}(G) \mid$.

If we choose $\epsilon$, such that $2^{1-\epsilon^{\prime}}<n^{\epsilon^{\prime}-\epsilon}$, then $\left|I^{*}(G)\right|<(n)^{\epsilon^{\prime}-\epsilon}(n)^{1-\epsilon^{\prime}} \mid I_{A L G}$ $(G)\left|=(n)^{1-\epsilon}\right| I_{A L G}(G) \mid$.

Hence, $\left|I^{*}(G)\right|<(n)^{1-\epsilon}\left|I_{A L G}(G)\right|$, which leads to a contradiction to Theorem 9. Therefore, the ACYCLIC MATChing PRoBlem cannot be approximated within a factor of $n^{1-\epsilon}$ for any $\epsilon>0$, unless $P=N P$.

\section{APX-Completeness}

In this section, we show that the ACYClic MATChing Problem is APXcomplete for $2 k+1$-regular graphs for $k \geq 3$, where $k$ is a constant.

To prove the result, we first show that the ACYCLIC MATChing Problem is approximable within a constant factor when restricted to $k$-regular graphs for $k \geq 3$, where $k$ is a constant. For the purpose, consider the following algorithm:

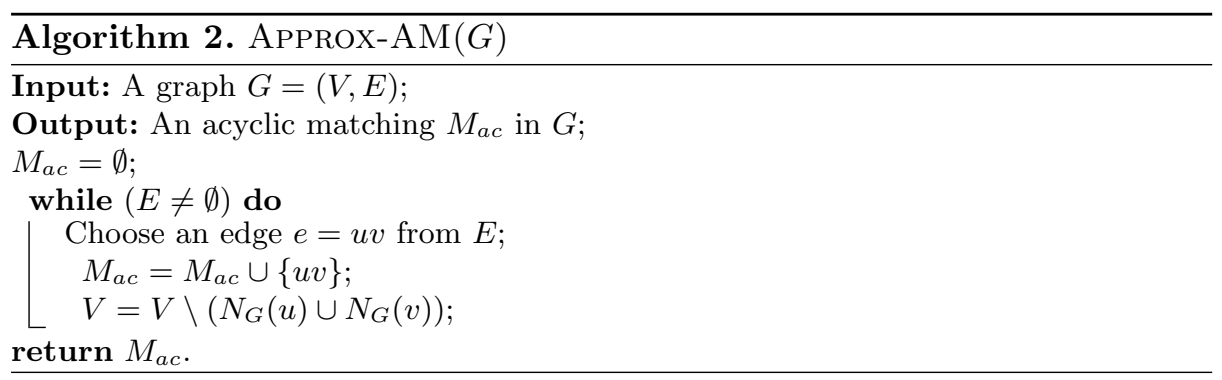

Lemma 8. The algorithm APPROX-AM(G) produces an acyclic matching of $G$ in polynomial time.

Proof. For any pair of edges in $M_{a c}$, say $e_{i}=a_{i} b_{i}$ and $e_{j}=a_{j} b_{j}, G\left[\left\{a_{i}, b_{i}, a_{j}, b_{j}\right\}\right]$ is a disjoint union of $K_{2}^{\prime} s$.

Lemma 9. The ACyclic MAtching Problem for a $k$-regular graph $G$ can be approximated with an approximation ratio of $\frac{2 k(k-1)+1}{k}$, where $k$ is a constant.

Proof. Given a $k$-regular graph $G$, construct an acyclic matching $M_{a c}$ of $G$ by using algorithm Approx-AM $(G)$. In each step, after adding an edge in the matching $M_{a c}$, we are removing at most $k^{2}$ edges, hence $\frac{k n}{2[2 k(k-1)+1]} \leq\left|M_{a c}\right|$. Moreover, it is easy to see that the size of any matching can be at most $\frac{n}{2}$.

Hence, the ACyclic Matching Problem is approximable within a factor of $\frac{2 k(k-1)+1}{k}$ in $k$-regular graphs, where $k$ is a constant. 
To prove the result, we will need the following theorem for the MAXIMUM INDEPENDENT SET PROBLEM.

Theorem 11. [1,10] The Maximum Independent Set Problem is APXcomplete for $k$-regular graphs for $k \geq 3$.

Observation 12. If $G$ is a k-regular graph in Construction 1 , then the constructed graph $H$ is a $2 k+1$-regular graph for $k \geq 3$.

Now, we are ready to prove the APX-completeness of the ACYCLIC MATCHING PROBLEM for $2 k+1$-regular graphs for $k \geq 3$, where $k$ is a constant. For this purpose, we recall the concept of $L$-reduction. Given two NP optimization problems $\pi_{1}$ and $\pi_{2}$ and a polynomial time transformation $f$ from instances of $\pi_{1}$ to instances of $\pi_{2}$, we say that $f$ is an $L$-reduction if there are positive constants $\alpha$ and $\beta$ such that for every instance $x$ of $\pi_{1}$ :

1. opt $_{\pi_{2}}(f(x)) \leq \alpha . o p t_{\pi_{1}}(x)$;

2. for every feasible solution $y$ of $f(x)$ with objective value $m_{\pi_{2}}(f(x), y)=c_{2}$, we can find a solution $y^{\prime}$ of $x$ in polynomial time with $m_{\pi_{1}}\left(x, y^{\prime}\right)=c_{1}$ such that $\left|o p t_{\pi_{1}}(x)-c_{1}\right| \leq \beta . \mid$ opt $_{\pi_{2}}(f(x))-c_{2} \mid$.

Theorem 13. The Acyclic Matching Problem is APX-complete for $2 k+1$ regular graphs for $k \geq 3$, where $k$ is a constant.

Proof. By Lemma 9, it is clear that the Acyclic Matching Problem for $2 k+$ 1-regular graphs for $k \geq 3$ belongs to the class APX. By Theorem 11, it is enough to construct an $L$-reduction from the instances of the MAXIMUM INDEPENDENT Set Problem for $k$-regular graphs to the instances of the ACYCLIC MATChing Problem for $2 k+1$-regular graphs. Given a $k$-regular graph $G=(V, E)$, where $V=\left\{v_{1}, v_{2}, \ldots, v_{n}\right\}$. We construct a graph $H=\left(V_{H}, E_{H}\right)$, an instance of the Acyclic Matching Problem by Construction 1. It is easy to see by Lemma 7 and Corollary 3 that the reduction described in Construction 1 is an L-reduction with $\alpha=1$ and $\beta=1$.

Therefore, the ACYClic MAtching Problem is APX-complete for $2 k+1$ regular graphs for $k \geq 3$, where $k$ is a constant.

\section{Conclusion}

In this paper, we have shown that the Acyclic Matching Decide Problem is NP-complete for comb-convex bipartite graphs and dually chordal graphs. On the positive side, we have shown that the Acyclic Matching Problem can be solved in linear time in split graphs and proper interval graphs. Apart from these, we have shown that the ACYCLIC MATCHING PROBLEM cannot be approximated within a factor of $n^{1-\epsilon}$ for any $\epsilon>0$, unless $P=N P$. We have also shown that the ACYClic MATChing Problem is APX-complete for $2 k+1$ regular graphs for $k \geq 3$, where $k$ is a constant. Further, it will be interesting to study better approximation algorithms for this problem for bipartite graphs and other important graph classes. 


\section{References}

1. Alimonti, P., Kann, V.: Some APX-completeness results for cubic graphs. Theoret. Comput. Sci. 237(1-2), 123-134 (2000)

2. Baste, J., Rautenbach, D.: Degenerate matchings and edge colorings. Discrete Appl. Math. 239, 38-44 (2018)

3. Fürst, M., Rautenbach, D.: A lower bound on the acyclic matching number of subcubic graphs. Discrete Math. 341(8), 2353-2358 (2018)

4. Fürst, M., Rautenbach, D.: On some hard and some tractable cases of the maximum acyclic matching problem. Ann. Oper. Res. 279(1), 291-300 (2019). https://doi. org/10.1007/s10479-019-03311-1

5. Goddard, W., Hedetniemi, S.M., Hedetniemi, S.T., Laskar, R.: Generalized subgraph-restricted matchings in graphs. Discrete Math. 293(1), 129-138 (2005)

6. Heggernes, P., Kratsch, D.: Linear-time certifying recognition algorithms and forbidden induced subgraphs. Nord. J. Comput. 14(1-2), 87-108 (2007)

7. Jamison, R.E., Laskar, R.: Elimination orderings of chordal graphs. In: Combinatorics and Applications, pp. 192-200 (1982)

8. Panda, B.S., Das, S.K.: A linear time recognition algorithm for proper interval graphs. Inf. Process. Lett. 87(3), 153-161 (2003)

9. Panda, B.S., Pradhan, D.: Acyclic matchings in subclasses of bipartite graphs. Discrete Math. Algorithms Appl. 4(04), 1250050 (2012)

10. Papadimitriou, C.H., Yannakakis, M.: Optimization, approximation, and complexity classes. J. Comput. Syst. Sci. 43(3), 425-440 (1991)

11. Bao, F.S., Zhang, Y.: A review of tree convex sets test. Comput. Intell. 28(3), 358-372 (2012)

12. Zuckerman, D.: Linear degree extractors and the inapproximability of max clique and chromatic number. In: Proceedings of the Thirty-Eighth Annual ACM Symposium on Theory of Computing, pp. 681-690 (2006) 